\title{
Tubular Surfaces Around Timelike Biharmonic Curves in Lorentzian Heisenberg Group $\mathrm{Heis}^{3}$
}

\author{
Talat KÖRPINAR and Essin TURHAN
}

\begin{abstract}
In this paper, we describe a new method for constructing a tubular surface surrounding a timelike biharmonic curve in the Lorentzian Heisenberg group $\mathrm{Heis}^{3}$. Firstly, we characterize timelike biharmonic curves in terms of their curvature and torsion. Also, by using timelike biharmonic curves, we give explicit parametrizations of tubular surfaces in the Lorentzian Heisenberg group $\mathrm{Heis}^{3}$.
\end{abstract}

\section{Introduction}

Tubular surfaces are among the surfaces which are easier to describe both analytically and "operationally". They are still under active investigation, both for finding best parameterizations (see, for instance, $[8,20]$ ) or for application in different fields (for instance in medicine, see [1])

We remind that, if $\mathrm{C}$ is a space curve, a tubular surface associated to this curve is a surface swept by a family of spheres of constant radius (which will be the radius of the tube), having the center on the given curve. Alternatively, as we shall see in the next section, for them we can construct quite easily a parameterization using the Frenet frame associate to the curve. The tubular surfaces are used quite often in computer graphics, but we think they deserve more attention for several reasons. For instance, there is the problem of representing the curves themselves. Usually, the space curves are represented by

Key Words: Tubular Surface,Biharmonic Curve, Heisenberg Group.

2010 Mathematics Subject Classification: 31B30, 58E20.

Received: July, 2010.

Accepted: August, 2011. 
using solids rather then tubes. There are, today, several very good computer algebra system (such as Maple, or Mathematica) which allow the vizualisation of curves and surfaces, in different kind of representations.

The aim of this paper is to study a tubular surface surrounding a timelike biharmonic curve in the Lorentzian Heisenberg group $\mathrm{Heis}^{3}$.

Firstly, harmonic maps are given as follows:

Let $\left(M^{m}, g\right)$ and $\left(N^{n}, h\right)$ be two Riemannian manifolds, the energy functional of a map $\phi \in C^{1}\left(M^{m}, N^{n}\right)$ is defined by

$$
E(\phi)=\frac{1}{2} \int_{M}|d \phi|^{2} d v_{g}
$$

where $|d \phi|$ is the Hilbert-Schmidt norm of the differential $d \phi$ and $d v_{g}$ is the volume element on $M$. A map $\phi \in C^{2}\left(M^{m}, N^{n}\right)$ is called harmonic if it is a critical point of the energy functional, that is, if it is a solution of the Euler-Lagrange equation associated to (1.1)

$$
\tau(\phi)=\operatorname{Tr}_{g} \nabla d \phi=0,
$$

$\tau(\phi)$ is called the tension field of $\phi$. Harmonic maps are solutions of a second order nonlinear elliptic system and they play a very important rôle in many branches of mathematics and physics where they may serve as a model for liquid crystal. One can refer to [9] for background on harmonic maps.

Secondly, biharmonic maps are given as follows:

A natural generalization of harmonic maps is given by integrating the square of the norm of the tension field. More precisely, the bi-energy functional of a map $\phi \in C^{2}\left(M^{m}, N^{n}\right)$ is defined by

$$
E_{2}(\phi)=\frac{1}{2} \int_{M}|\tau(\phi)|^{2} d v_{g}
$$

a map $\phi \in C^{4}\left(M^{m}, N^{n}\right)$ is called biharmonic if it is a critical point of the bi-energy functional, that is, if it is a solution of the Euler-Lagrange equation associated to (1.3)

$$
\tau_{2}(\phi)=-\operatorname{Tr}_{g}\left(\nabla^{\phi}\right)^{2} \tau(\phi)-\operatorname{Tr}_{g} R^{N}(\tau(\phi), d \phi) d \phi=0,
$$

where

$$
\operatorname{Tr}_{g}\left(\nabla^{\phi}\right)^{2}=\sum_{i=1}^{m}\left(\nabla_{e_{i}}^{\phi} \nabla_{e_{i}}^{\phi}-\nabla_{\nabla_{e_{i}}^{M} e_{i}}^{\phi}\right)
$$

for an orthonormal frame $\left\{e_{1}, e_{2}, . ., e_{m}\right\}$, is the Laplacian on sections of the pull-back bundle $\phi^{-1} T N$ and $R^{N}$ is the curvature operator on $N$. 
Biharmonic functions are utilized in many physical situations, particularly in fluid dynamics and elasticity problems. Most important applications of the theory of functions of a complex variable were obtained in the plane theory of elasticity and in the approximate theory of plates subject to normal loading. That is, in cases when the solutions are biharmonic functions or functions associated with them. In linear elasticity, if the equations are formulated in terms of displacements for two-dimensional problems then the introduction of a stress function leads to a fourth-order equation of biharmonic type. For instance, the stress function is proved to be biharmonic for an elastically isotropic crystal undergoing phase transition, which follows spontaneous dilatation. Biharmonic functions also arise when dealing with transverse displacements of plates and shells. They can describe the deflection of a thin plate subjected to uniform loading over its surface with fixed edges. Biharmonic functions arise in fluid dynamics, particularly in Stokes flow problems (i.e., low-Reynolds-number flows). There are many applications for Stokes flow such as in engineering and biological transport phenomena (for details, see $[8,13])$. Fluid flow through a narrow pipe or channel, such as that used in micro-fluidics, involves low Reynolds number. Seepage flow through cracks and pulmonary alveolar blood flow can also be approximated by Stokes flow. Stokes flow also arises in flow through porous media, which have been long applied by civil engineers to groundwater movement. The industrial applications include the fabrication of microelectronic components, the effect of surface roughness on lubrication, the design of polymer dies and the development of peristaltic pumps for sensitive viscous materials. In natural systems, creeping flows are important in biomedical applications and studies of animal locomotion.

In [3] the authors completely classified the biharmonic submanifolds of the three-dimensional sphere, while in [4] there were given new methods to construct biharmonic submanifolds of codimension greater than one in the ndimensional sphere. The biharmonic submanifolds into a space of nonconstant sectional curvature were also investigated. The proper biharmonic curves on Riemannian surfaces were studied in [5]. Inoguchi classified the biharmonic Legendre curves and the Hopf cylinders in three-dimensional Sasakian space forms [9]. Then, Sasahara gave in [19] the explicit representation of the proper biharmonic Legendre surfaces in five-dimensional Sasakian space forms.

In this paper, we describe a new method for constructing a tubular surface surrounding a timelike biharmonic curve in the Lorentzian Heisenberg group $H_{e i s^{3}}$. Firstly, we characterize timelike biharmonic curves in terms of their curvature and torsion. Also, by using timelike biharmonic curves, we give explicit parametrizations of tubular surface in the Lorentzian Heisenberg group $\mathrm{Heis}^{3}$. 


\section{The Lorentzian Heisenberg Group Heis ${ }^{3}$}

Heisenberg group plays an important role in many branches of mathematics such as representation theory, harmonic analysis, PDEs or even quantum mechanics, where it was initially defined as a group of $3 \times 3$ matrices

$$
\left\{\left(\begin{array}{ccc}
1 & x & z \\
0 & 1 & y \\
0 & 0 & 1
\end{array}\right): x, y, z \in \mathbb{R}\right\}
$$

with the usual multiplication rule.

We will use the following complex definition of the Heisenberg group.

$$
\text { Heis }^{3}=\mathbb{C} \times \mathbb{R}=\{(w, z): w \in \mathbb{C}, z \in \mathbb{R}\}
$$

with

$$
(w, z) *(\bar{w}, \bar{z})=(w+\bar{w}, z+\bar{z}+\Im(\langle w, \bar{w}\rangle)),
$$

where $\langle$,$\rangle is the usual Hermitian product in \mathbb{C}$.

The identity of the group is $(0,0,0)$ and the inverse of $(x, y, z)$ is given by $(-x,-y,-z)$.

Let $a=\left(w_{1}, z_{1}\right), b=\left(w_{2}, z_{2}\right)$ and $c=\left(w_{3}, z_{3}\right)$. The commutator of the elements $a, b \in \mathrm{Heis}^{3}$ is equal to

$$
\begin{aligned}
{[a, b] } & =a * b * a^{-1} * b^{-1} \\
& =\left(w_{1}, z_{1}\right) *\left(w_{2}, z_{2}\right) *\left(-w_{1},-z_{1}\right) *\left(-w_{2},-z_{2}\right) \\
& =\left(w_{1}+w_{2}-w_{1}-w_{2}, z_{1}+z_{2}-z_{1}-z_{2}\right) \\
& =(0, \alpha)
\end{aligned}
$$

where $\alpha \neq 0$ in general. For example

$$
[(1,0),(i, 0)]=(0,2) \neq(0,0) .
$$

Which shows that $\mathrm{Heis}^{3}$ is not abelian.

On the other hand, for any $a, b, c \in \mathrm{Heis}^{3}$, their double commutator is

$$
\begin{aligned}
{[[a, b], c] } & =\left[(0, \alpha),\left(w_{3}, z_{3}\right)\right] \\
& =(0,0) .
\end{aligned}
$$

This implies that $\mathrm{Heis}^{3}$ is a nilpotent Lie group with nilpotency 2.

The left-invariant Lorentz metric on $\mathrm{Heis}^{3}$ is

$$
g=-d x^{2}+d y^{2}+(x d y+d z)^{2} .
$$


The following set of left-invariant vector fields forms an orthonormal basis for the corresponding Lie algebra:

$$
\left\{\mathbf{e}_{1}=\frac{\partial}{\partial z}, \mathbf{e}_{2}=\frac{\partial}{\partial y}-x \frac{\partial}{\partial z}, \mathbf{e}_{3}=\frac{\partial}{\partial x}\right\}
$$

The characterising properties of this algebra are the following commutation relations:

$$
\left[\mathbf{e}_{2}, \mathbf{e}_{3}\right]=2 \mathbf{e}_{1}, \quad\left[\mathbf{e}_{3}, \mathbf{e}_{1}\right]=0, \quad\left[\mathbf{e}_{2}, \mathbf{e}_{1}\right]=0,
$$

with

$$
g\left(\mathbf{e}_{1}, \mathbf{e}_{1}\right)=g\left(\mathbf{e}_{2}, \mathbf{e}_{2}\right)=1, \quad g\left(\mathbf{e}_{3}, \mathbf{e}_{3}\right)=-1 .
$$

Proposition 2.1. For the covariant derivatives of the Levi-Civita connection of the left-invariant metric $g$, defined above the following is true:

$$
\nabla=\left(\begin{array}{ccc}
\mathbf{0} & \mathbf{e}_{3} & \mathbf{e}_{2} \\
\mathbf{e}_{3} & \mathbf{0} & \mathbf{e}_{1} \\
\mathbf{e}_{2} & -\mathbf{e}_{1} & \mathbf{0}
\end{array}\right)
$$

where the $(i, j)$-element in the table above equals $\nabla_{e_{i}} e_{j}$ for our basis

$$
\left\{\mathbf{e}_{k}, k=1,2,3\right\}=\left\{\mathbf{e}_{1}, \mathbf{e}_{2}, \mathbf{e}_{3}\right\} .
$$

We adopt the following notation and sign convention for Riemannian curvature operator:

$$
R(X, Y) Z=-\nabla_{X} \nabla_{Y} Z+\nabla_{Y} \nabla_{X} Z+\nabla_{[X, Y]} Z
$$

The Riemannian curvature tensor is given by

$$
R(X, Y, Z, W)=g(R(X, Y) Z, W) .
$$

Moreover we put

$$
R_{i j k}=R\left(\mathbf{e}_{i}, \mathbf{e}_{j}\right) \mathbf{e}_{k}, \quad R_{i j k l}=R\left(\mathbf{e}_{i}, \mathbf{e}_{j}, \mathbf{e}_{k}, \mathbf{e}_{l}\right),
$$

where the indices $i, j, k$ and $l$ take the values 1,2 and 3 .

$$
R_{121}=-\mathbf{e}_{2}, \quad R_{131}=-\mathbf{e}_{3}, \quad R_{232}=3 \mathbf{e}_{3}
$$

and

$$
R_{1212}=-1, \quad R_{1313}=1, \quad R_{2323}=-3 .
$$




\section{Timelike Biharmonic Curves in the Lorentzian Heisen- berg Group Heis ${ }^{3}$}

An arbitrary curve $\gamma: I \longrightarrow \mathrm{Heis}^{3}$ is spacelike, timelike or null, if all of its velocity vectors $\gamma^{\prime}(s)$ are, respectively, spacelike, timelike or null, for each $s \in I \subset \mathbb{R}$. Let $\gamma: I \longrightarrow$ Heis $^{3}$ be a unit speed timelike curve and $\{\mathbf{T}, \mathbf{N}, \mathbf{B}\}$ are Frenet vector fields, then Frenet formulas are as follows

$$
\begin{aligned}
\nabla_{\mathbf{T}} \mathbf{T} & =\kappa_{1} \mathbf{N}, \\
\nabla_{\mathbf{T}} \mathbf{N} & =\kappa_{1} \mathbf{T}+\kappa_{2} \mathbf{B}, 3.1 \\
\nabla_{\mathbf{T}} \mathbf{B} & =-\kappa_{2} \mathbf{N},
\end{aligned}
$$

where $\kappa_{1}, \kappa_{2}$ are curvature function and torsion function, respectively.

With respect to the orthonormal basis $\left\{\mathbf{e}_{1}, \mathbf{e}_{2}, \mathbf{e}_{3}\right\}$ we can write

$$
\begin{aligned}
\mathbf{T} & =T_{1} \mathbf{e}_{1}+T_{2} \mathbf{e}_{2}+T_{3} \mathbf{e}_{3}, \\
\mathbf{N} & =N_{1} \mathbf{e}_{1}+N_{2} \mathbf{e}_{2}+N_{3} \mathbf{e}_{3}, \\
\mathbf{B} & =\mathbf{T} \times \mathbf{N}=B_{1} \mathbf{e}_{1}+B_{2} \mathbf{e}_{2}+B_{3} \mathbf{e}_{3} .
\end{aligned}
$$

Theorem 3.1. $\gamma: I \longrightarrow \mathrm{Heis}^{3}$ is a unit speed timelike biharmonic curve if and only if

$$
\begin{aligned}
\kappa_{1} & =\text { constant } \neq 0, \\
\kappa_{1}^{2}-\kappa_{2}^{2} & =1-4 B_{1}^{2}, 3.2 \\
\kappa_{2}^{\prime} & =2 N_{1} B_{1} .
\end{aligned}
$$

Proof. Using (3.1), we see that $\gamma$ is a unit speed timelike biharmonic curve if and only if

$$
\begin{aligned}
\kappa_{1} \kappa_{1}^{\prime} & =0 \\
\kappa_{1}^{\prime \prime}+\kappa_{1}^{3}-\kappa_{1} \kappa_{2}^{2} & =\kappa_{1} R(\mathbf{T}, \mathbf{N}, \mathbf{T}, \mathbf{N}), 3.3 \\
2 \kappa_{2} \kappa_{1}^{\prime}+\kappa_{2}^{\prime} \kappa_{1} & =\kappa_{1} R(\mathbf{T}, \mathbf{N}, \mathbf{T}, \mathbf{B}) .
\end{aligned}
$$

Since $\kappa_{1} \neq 0$ by the assumption that is non-geodesic

$$
\begin{aligned}
\kappa_{1} & =\text { constant } \neq 0, \\
\kappa_{1}^{2}-\kappa_{2}^{2} & =R(\mathbf{T}, \mathbf{N}, \mathbf{T}, \mathbf{N}), 3.4 \\
\kappa_{2}^{\prime} & =R(\mathbf{T}, \mathbf{N}, \mathbf{T}, \mathbf{B}) .
\end{aligned}
$$


A direct computation using (2.4) yields

$$
\begin{aligned}
R(\mathbf{T}, \mathbf{N}, \mathbf{T}, \mathbf{N}) & =1-4 B_{1}^{2}, 3.5 \\
R(\mathbf{T}, \mathbf{N}, \mathbf{T}, \mathbf{B}) & =2 N_{1} B_{1} .3 .6
\end{aligned}
$$

These, together with (3.4), complete the proof of the theorem.

Theorem 3.2. If $\gamma: I \longrightarrow$ Heis $^{3}$ is a unit speed timelike biharmonic curve, then $\gamma$ is a timelike helix.

Proof. We can use (2.3) to compute the covariant derivatives of the vector fields $\mathbf{T}, \mathbf{N}$ and $\mathbf{B}$ as:

$$
\begin{aligned}
& <\nabla_{\mathbf{T}} \mathbf{T}, \mathbf{e}_{1}>=T_{1}^{\prime}, \\
& <\nabla_{\mathbf{T}} \mathbf{N}, \mathbf{e}_{1}>=N_{1}^{\prime}+T_{2} N_{3}-T_{3} N_{2}, 3.7 \\
& <\nabla_{\mathbf{T}} \mathbf{B}, \mathbf{e}_{1}>=B_{1}^{\prime}+T_{2} B_{3}-T_{3} B_{2} .
\end{aligned}
$$

On the other hand, using Frenet formulas (3.1), we have

$$
\begin{aligned}
& <\nabla_{\mathbf{T}} \mathbf{T}, \mathbf{e}_{1}>=\kappa_{1} N_{1}, \\
& <\nabla_{\mathbf{T}} \mathbf{N}, \mathbf{e}_{1}>=\kappa_{1} T_{1}+\kappa_{2} B_{1}, 3.8 \\
& <\nabla_{\mathbf{T}} \mathbf{B}, \mathbf{e}_{1}>=-\kappa_{2} N_{1} .
\end{aligned}
$$

These, together with (3.7) and (3.8), give

$$
\begin{aligned}
T_{1}^{\prime} & =\kappa_{1} N_{1}, \\
N_{1}^{\prime}+T_{2} N_{3}-T_{3} N_{2} & =\kappa_{1} T_{1}+\kappa_{2} B_{1}, 3.9 \\
B_{1}^{\prime}+T_{2} B_{3}-T_{3} B_{2} & =-\kappa_{2} N_{1} .
\end{aligned}
$$

Assume that $\gamma$ is biharmonic. Then using $\kappa_{2}^{\prime}=2 N_{1} B_{1} \neq 0$ and (3.2), we obtain

$$
-2 \kappa_{2}^{\prime} \kappa_{2}=8 B_{1} B_{1}^{\prime}
$$

and

$$
\kappa_{2} N_{1} B_{1}=2 B_{1} B_{1}^{\prime} .
$$

Then,

$$
\kappa_{2}=\frac{2 B_{1}^{\prime}}{N_{1}} .
$$

If we use $T_{2} B_{3}-T_{3} B_{2}=N_{1}$ and (3.9), we get

$$
B_{1}^{\prime}=\left(1-\kappa_{2}\right) N_{1} .
$$


We substitute $B_{1}^{\prime}$ in equation (3.10):

$$
\kappa_{2}=\frac{2}{3}=\text { constant. }
$$

Therefore, also $\kappa_{2}$ is constant and we have a contradiction that is $\kappa_{2}^{\prime}=$ $N_{1} B_{1} \neq 0$. This completes the proof.

Corollary 3.3. $\gamma: I \longrightarrow$ Heis $^{3}$ is a unit speed timelike biharmonic if and only if

$$
\begin{aligned}
\kappa_{1} & =\text { constant } \neq 0, \\
\kappa_{2} & =\text { constant, } 3.11 \\
N_{1} B_{1} & =0, \\
\kappa_{1}^{2}-\kappa_{2}^{2} & =1-4 B_{1}^{2} .
\end{aligned}
$$

\section{Tubular Surfaces In The Lorentzian Heisenberg Group $\mathrm{Heis}^{3}$}

We shall give here the mathematical description of tubular surfaces associated to timelike biharmonic curves in the Lorentzian Heisenberg group Heis ${ }^{3}$. Our purpose in this section, we will obtain the tubular surface from the canal surface in the Lorentzian Heisenberg group $\mathrm{Heis}^{3}$. If we find the canal surface with taking variable radius $r(s)$ as constant, then the tubular surface can be found, since the canal surface is a general case of the tubular surface. An envelope of a 1-parameter family of surfaces is constructed in the same way that we constructed a 1-parameter family of curves. The family is described by a differentiable function $F(x, y, z, \lambda)=0$, where $\lambda$ is a parameter. When $\lambda$ can be eliminated from the equations

$$
F(x, y, z, \lambda)=0
$$

and

$$
\frac{\partial F(x, y, z, \lambda)}{\partial \lambda}=0 .
$$

We get the envelope,which is a surface described implicitly as $G(x, y, z)=$ 0 . For example, for a 1-parameter family of planes we get a develople surface $[7]$. 
Definition 4.1. The envelope of a 1-parameter family of the Lorentzian spheres in the Lorentzian Heisenberg group Heis ${ }^{3}$ is called a canal surface in the Lorentzian Heisenberg group $\mathrm{Heis}^{3}$. The curve formed by the centers of the Lorentzian spheres is called center curve of the canal surface. The radius of the canal surface is the function $r$ such that $r(s)$ is the radius of the Lorentzian sphere.

Definition 4.2. Let $\gamma: I \longrightarrow \mathrm{Heis}^{3}$ be a unit speed curve whose curvature does not vanish. Consider a tube of radius $r$ around $\gamma$. Since the normal $N$ and binormal $B$ are perpendicular to $\gamma$, the Lorentzian circle is perpendicular $\gamma$ and $\gamma(s)$. As this Lorentzian circle moves along $\gamma$, it traces out a surface about $\gamma$ which will be the tube about $\gamma$, provided $r$ is not too large.

It is easy to see that when the radius function $r(s)$ is constant, the definition of canal surface reduces to the definition of a tube. In fact, we can characterize tubes among all canal surfaces.

Theorem 4.3. Let the center curve of a tubular surface $C(s, t)$ is a unit speed timelike biharmonic curve $\gamma: I \longrightarrow$ Heis $^{3}$. Then, the parametric equations of $C(s, t)$ are

$$
\begin{aligned}
x(s, t)= & \frac{1}{\wp} \cosh \varphi \sinh (\wp s+\zeta)+\frac{r}{\kappa_{1}} \cosh \varphi(\wp+2 \sinh \varphi) \\
& \sinh (\wp s+\zeta) \cos t+\frac{r}{\kappa_{1}} \cosh \varphi(\wp+2 \sinh \varphi)(\cosh \varphi \\
& \sinh (\wp s+\zeta)\left[-\frac{1}{\wp^{2}} \sinh (\wp s+\zeta) \cosh (\wp s+\zeta)\right. \\
& \left.-c_{1} s \cosh (\wp s+\zeta)-c_{2} \cosh (\wp s+\zeta)\right]-\cosh (\wp s+\zeta)[\sinh \varphi \\
& \left.\left.-\frac{1}{\wp} \cosh ^{2} \varphi \sinh ^{2}(\wp s+\zeta)-a_{1} \cosh \varphi \sinh (\wp s+\zeta)\right]\right) \sin t+a_{1}, \\
y(s, t)= & \frac{1}{\wp} \cosh \varphi \cosh (\wp s+\zeta)+\frac{r}{\kappa_{1}} \cosh \varphi(\wp+2 \sinh \varphi) \\
& \cosh (\wp s+\zeta) \cos t+\frac{r}{\kappa_{1}} \cosh \varphi(\wp+2 \sinh \varphi)[\sinh (\wp s+\zeta) 4 .(111) \\
& {\left[\sinh \varphi-\frac{1}{\wp} \cosh ^{2} \varphi \sinh ^{2}(\wp s+\zeta)-a_{1} \cosh \varphi \sinh (\wp s+\zeta)\right] } \\
& -\cosh \varphi \cosh (\wp s+\zeta)\left[-\frac{1}{\wp^{2}} \sinh (\wp s+\zeta) \cosh (\wp s+\zeta)\right. \\
& \left.-c_{1} s \cosh (\wp s+\zeta)-c_{2} \cosh (\wp s+\zeta)\right] \sin t+a_{2},
\end{aligned}
$$




$$
\begin{aligned}
z(s, t)= & {\left[\sinh \varphi+\frac{1}{\wp} \cosh ^{2} \varphi\right] s-\frac{1}{4 \wp^{2}} \cosh ^{2} \varphi \sinh 2(\wp s+\zeta) } \\
& -\frac{a_{1}}{\wp} \cosh ^{2}(\wp s+\zeta)+\frac{r}{\kappa_{1}} \cosh \varphi(\wp+2 \sinh \varphi) \\
& \left(-\frac{1}{\wp^{2}} \sinh (\wp s+\zeta) \cosh (\wp s+\zeta)-c_{1} s \cosh (\wp s+\zeta)\right. \\
& \left.-c_{2} \cosh (\wp s+\zeta)\right) \cos t+\frac{r}{\kappa_{1}} \cosh \varphi(\wp+2 \sinh \varphi)(\cosh \varphi \\
& \left.\cosh ^{2}(\wp s+\zeta)-\cosh \varphi \sinh ^{2}(\wp s+\zeta)\right) \sin t+a_{3},
\end{aligned}
$$

where $\wp=\frac{\kappa_{1}-\sinh 2 \varphi}{\cosh \varphi}$ and $a_{1}, a_{2}, a_{3}, c_{1}, c_{2}$ are constants of integration.

Proof. Assume that the center curve of a tubular surface $C(s, t)$ is a unit speed timelike biharmonic curve $\gamma$ and $C$ denote a patch that parametrizes the envelope of the Lorentzian spheres defining the tubular surface. Then we obtain

$$
C(s, t)=\gamma(s)+\xi(s, t) \mathbf{T}+\eta(s, t) \mathbf{N}+\rho(s, t) \mathbf{B},
$$

where $\xi, \eta$ and $\rho$ are differentiable on the interval on which $\gamma$ is defined.

Since $\gamma$ is timelike biharmonic, $\gamma$ is a timelike helix. So, without loss of generality, we take the axis of $\gamma$ is parallel to the spacelike vector $\mathbf{e}_{1}$. Then,

$$
g\left(\mathbf{T}, \mathbf{e}_{1}\right)=T_{1}=\sinh \varphi,
$$

where $\varphi$ is constant angle.

The tangent vector can be written in the following form

$$
\mathbf{T}=T_{1} \mathbf{e}_{1}+T_{2} \mathbf{e}_{2}+T_{3} \mathbf{e}_{3} .
$$

On the other hand, the tangent vector $\mathbf{T}$ is a unit timelike vector, so the following condition is satisfied

$$
T_{2}^{2}-T_{3}^{2}=-1-\sinh ^{2} \varphi .
$$

Noting that $\cosh ^{2} \varphi-\sinh ^{2} \varphi=1$, we have

$$
T_{3}^{2}-T_{2}^{2}=\cosh ^{2} \varphi
$$

The general solution of (4.5) can be written in the following form

$$
\begin{aligned}
& T_{2}=\cosh \varphi \sinh \mu, 4.6 \\
& T_{3}=\cosh \varphi \cosh \mu
\end{aligned}
$$

where $\mu$ is an arbitrary function of $s$. 
So, substituting the components $T_{1}, T_{2}$ and $T_{3}$ in the equation (4.3), we have the following equation

$$
\mathbf{T}=\sinh \varphi \mathbf{e}_{1}+\cosh \varphi \sinh \mu \mathbf{e}_{2}+\cosh \varphi \cosh \mu \mathbf{e}_{3} .
$$

Since $\left|\nabla_{\mathbf{T}} \mathbf{T}\right|=\kappa_{1}$, we obtain

$$
\mu=\left(\frac{\kappa_{1}-\sinh 2 \varphi}{\cosh \varphi}\right) s+\zeta
$$

where $\zeta \in \mathbb{R}$.

Thus (4.7) and (4.8), imply

$$
\mathbf{T}=\sinh \varphi \mathbf{e}_{1}+\cosh \varphi \sinh (\wp s+\zeta) \mathbf{e}_{2}+\cosh \varphi \cosh (\wp s+\zeta) \mathbf{e}_{3},
$$

where $\wp=\frac{\kappa_{1}-\sinh 2 \varphi}{\cosh \varphi}$

Using (2.1) in (4.8), we obtain

$$
\begin{aligned}
\mathbf{T}= & (\cosh \varphi \cosh (\wp s+\zeta), \cosh \varphi \sinh (\wp s+\zeta), \sinh \varphi 4.10 \\
& \left.-\frac{1}{\wp} \cosh ^{2} \varphi \sinh ^{2}(\wp s+\zeta)-a_{1} \cosh \varphi \sinh (\wp s+\zeta)\right),
\end{aligned}
$$

where $a_{1}$ is constant of integration.

Now, taking into account (4.10), we obtain

$$
\begin{aligned}
x(s)= & \frac{1}{\wp} \cosh \varphi \sinh (\wp s+\zeta)+a_{1}, \\
y(s)= & \frac{1}{\wp} \cosh \varphi \cosh (\wp s+\zeta)+a_{2}, 4.11 \\
z(s)= & {\left[\sinh \varphi+\frac{1}{\wp} \cosh ^{2} \varphi\right] s-\frac{1}{4 \wp^{2}} \cosh ^{2} \varphi \sinh 2(\wp s+\zeta) } \\
& -\frac{a_{1}}{\wp} \cosh ^{2}(\wp s+\zeta)+a_{3},
\end{aligned}
$$

where $a_{1}, a_{2}, a_{3}$ are constants of integration.

On the other hand, using Frenet formulas (3.1) and (4.9), we have

$$
\begin{aligned}
\mathbf{N}= & \frac{1}{\kappa_{1}}\left[\cosh \varphi \cosh (\wp s+\zeta)(\wp+2 \sinh \varphi) \mathbf{e}_{2} 4.12\right. \\
& \left.+\cosh \varphi \sinh (\wp s+\zeta)(\wp+2 \sinh \varphi) \mathbf{e}_{3}\right]
\end{aligned}
$$


Smilarly, using (2.1) in (4.12), we obtain

$$
\begin{aligned}
\mathbf{N}= & \frac{1}{\kappa_{1}} \cosh \varphi(\wp+2 \sinh \varphi)(\sinh (\wp s+\zeta), \cosh (\wp s+\zeta), \\
& -\frac{1}{\wp^{2}} \sinh (\wp s+\zeta) \cosh (\wp s+\zeta) 4.13 \\
& \left.-c_{1} s \cosh (\wp s+\zeta)-c_{2} \cosh (\wp s+\zeta)\right) .
\end{aligned}
$$

Hence,using (2.1), (4.9) and (4.12) then the binormal vector $\mathbf{B}$ is

$$
\begin{aligned}
\mathbf{B}= & \frac{1}{\kappa_{1}} \cosh \varphi(\wp+2 \sinh \varphi)(\cosh \varphi \sinh (\wp s+\zeta) \\
& {\left[-\frac{1}{\wp^{2}} \sinh (\wp s+\zeta) \cosh (\wp s+\zeta)-c_{1} s \cosh (\wp s+\zeta)\right.} \\
& \left.-c_{2} \cosh (\wp s+\zeta)\right]-\cosh (\wp s+\zeta)[\sinh \varphi \\
& \left.-\frac{1}{\wp} \cosh ^{2} \varphi \sinh ^{2}(\wp s+\zeta)-a_{1} \cosh \varphi \sinh (\wp s+\zeta)\right], 4.14 \\
& \sinh (\wp s+\zeta)\left[\sinh \varphi-\frac{1}{\wp} \cosh ^{2} \varphi \sinh ^{2}(\wp s+\zeta)\right. \\
& \left.-a_{1} \cosh \varphi \sinh (\wp s+\zeta)\right]-\cosh \varphi \cosh (\wp s+\zeta) \\
& {\left[-\frac{1}{\wp^{2}} \sinh (\wp s+\zeta) \cosh (\wp s+\zeta)-c_{1} s \cosh (\wp s+\zeta)\right.} \\
& \left.\left.-c_{2} \cosh (\wp s+\zeta)\right], \cosh \varphi \cosh ^{2}(\wp s+\zeta)-\cosh \varphi \sinh ^{2}(\wp s+\zeta)\right) .
\end{aligned}
$$

Using Definition 4.1 we have

$$
g(C(s, t)-\gamma(s), C(s, t)-\gamma(s))=r^{2} .
$$

Since $C(s, t)-\gamma(s)$ is a normal vector to the tubular surface, we get

$$
g\left(C(s, t)-\gamma(s), C_{s}(s, t)\right)=0 .
$$

From (4.2) and (4.15), we get

$$
\begin{aligned}
-\xi^{2}+\eta^{2}+\rho^{2} & =r^{2}, 4.17 \\
-\xi \xi_{s}+\eta \eta_{s}+\rho \rho_{s} & =0 .
\end{aligned}
$$

When we differentiate (4.17) with respect to $s$ and use the Frenet-Serret formulas, we obtain

$$
C_{s}=\left(1+\xi_{s}+\eta \kappa_{1}\right) \mathbf{T}+\left(\xi \kappa_{1}-\rho \kappa_{2}+\eta_{s}\right) \mathbf{N}+\left(\rho_{s}+\eta \kappa_{2}\right) \mathbf{B} .
$$


Then (4.16), (4.17), (4.18) and (4.19) imply that

$$
\xi=0 .
$$

Also, from (4.17) and (4.18) we get

$$
\eta^{2}+\rho^{2}=r^{2} .
$$

The solution of (4.20) can be written in the following form

$$
\begin{aligned}
\eta & =r \cos t, 4.21 \\
\rho & =r \sin t .
\end{aligned}
$$

Thus (4.2) becomes

$$
C(s, t)=\gamma(s)+r \mathbf{N}(s) \cos t+r \mathbf{B}(s) \sin t .
$$

Substuting (4.13), (4.14) and (4.11) into (4.22) we obtain the system (4.1). This completes the proof.

\section{References}

[1] A. Bornik, B. Reitinger, R. Beichel, Simplex-Mesh based Surface Reconstruction and Representation of Tubular Structures, in Proceedings of BVM2005, Springer, (2005).

[2] A.V. Bäcklund, Einiges über Curven- und Flächen-Transformationen, Lunds Univ. Arsskr. 10 (1874), 1-12.

[3] R. Caddeo, C. Oniciuc, P. Piu, Explicit formulas for non-geodesic biharmonic curves of the Heisenberg group, Rend. Sem. Mat. Univ. Politec. Torino 62 (3) (2004), 265-277.

[4] R. Caddeo, S. Montaldo, C. Oniciuc, Biharmonic submanifolds in spheres, Israel J. Math. 130 (2002), 109-123.

[5] R. Caddeo, S. Montaldo, P. Piu, Biharmonic curves on a surface, Rend. Mat. Appl. 21 (2001), 143-157.

[6] J. Eells, J.H. Sampson, Harmonic mappings of Riemannian manifolds, Amer. J. Math. 86 (1964), 109-160.

[7] A. Gray, Modern Differential Geometry of Curves and Surfaces with Mathematica, CRC Press, 1998 
[8] J. Happel, H. Brenner, Low Reynolds Number Hydrodynamics with Special Applications to Particulate Media, Prentice-Hall, New Jersey, (1965)

[9] J. Inoguchi, Submanifolds with harmonic mean curvature in contact 3manifolds, Colloq. Math. 100 (2004), 163-179.

[10] G.Y. Jiang, 2-harmonic maps and their first and second variation formulas, Chinese Ann. Math. Ser. A 7 (1986), 389-402.

[11] T. Körpınar, E. Turhan, On Horizontal Biharmonic Curves In The Heisenberg Group Heis ${ }^{3}$, Arab. J. Sci. Eng. Sect. A Sci. 35 (1) (2010), 79-85.

[12] G. Landsmann, J. Schicho, F. Winkler, The Parametrization of Canal Surfaces and the Decomposition of Polynomials into a Sum of Two Squares, J. Symb. Comput. 32(1/2)(2001), 119-132.

[13] W. E. Langlois, Slow Viscous Flow, Macmillan, New York, CollierMacmillan, London, 1964.

[14] V.B. Matveev , Salle M.A., Darboux transformations and solitons, Springer Series in Nonlinear Dynamics, Springer, Berlin, 1991.

[15] A. V. Mikharlov, A. B. Shabat, V. V. Sokolov, The symmetry approach to classification of integrable equations. What Is Integrability? Springer Series on Nonlinear Dynamics, Springer-Verlag, Berlin (1991), 115-184.

[16] C. Oniciuc, On the second variation formula for biharmonic maps to a sphere, Publ. Math. Debrecen 61 (2002), 613-622.

[17] Y. L. Ou, p-Harmonic morphisms, biharmonic morphisms, and nonharmonic biharmonic maps, J. Geom. Phys. 56 (2006), 358-374.

[18] S. Rahmani, Metriqus de Lorentz sur les groupes de Lie unimodulaires, de dimension trois, Journal of Geometry and Physics 9 (1992), 295-302.

[19] T. Sasahara, Legendre surfaces in Sasakian space forms whose mean curvature vectors are eigenvectors, Publ. Math. Debrecen 67 (2005), 285-303.

[20] J. Schicho, Proper Parametrization of Real Tubular Surfaces, J. Symb. Comput. 30(5) (2000), 583-593.

[21] E. Turhan, T. Körpmar, Position vector of spacelike biharmonic curves in the Lorentzian Heisenberg group Heis ${ }^{3}$, An. St. Univ. Ovidius Constanta 19 (1) (2011), 285-296. 
[22] E. Turhan, T. Körpınar, Characterize on the Heisenberg Group with left invariant Lorentzian metric, Demonstratio Mathematica 42 (2) (2009), 423-428.

[23] E. Turhan, T. Körpınar, On Characterization Of Timelike Horizontal Biharmonic Curves In The Lorentzian Heisenberg Group Heis ${ }^{3}$, Zeitschrift für Naturforschung A- A Journal of Physical Sciences 65a (2010), 641-648.

Talat KÖRPINAR,

Firat University,

Department of Mathematics, 23119, Elazı̆̆,

TURKEY

e-mail: talatkorpinar@gmail.com

Essin TURHAN,

Firat University,

Department of Mathematics, 23119, Elazı̆̆,

TURKEY

e-mail: talatkorpinar@gmail.com 
